OnLine Journal of Biological Sciences 11 (4): 105-111, 2011

ISSN 1608-4217

(C) 2011 A. Pandey et al., This open access article is distributed under a Creative Commons Attribution

(CC-BY) 3.0 license ns

\title{
Assessment of Genetic Diversity among Sugarcane Cultivars (Saccharum officinarum L.) using Simple Sequence Repeats Markers
}

\author{
${ }^{1}$ Ashutosh Pandey, ${ }^{2}$ Rupesh K. Mishra, \\ ${ }^{3}$ Shashank Mishra, ${ }^{3}$ Yogendra P. Singh and ${ }^{3}$ Sailesh Pathak \\ ${ }^{1}$ Center for Biotechnology, School of Environmental Biology, \\ A.P.S. University, Rewa-486 003, Madhya Pradesh, India \\ ${ }^{2}$ Department of Biotechnology, Integral University, \\ Kursi Road Lucknow-226 026, Uttar Pradesh, India \\ ${ }^{3}$ Department of Biotechnology, Dayanand Bachhrawan PG College, \\ Bachhrawan, Raebareli-229 301, Uttar Pradesh, India
}

\begin{abstract}
Problem statement: Increasing sugar productivity is the main concern of sugarcane (Saccharum officinarum L.) breeding programs. The complexity and size of the sugarcane genome is a major limitation in its genetic improvement. Characterization of sugarcane provides essential information of genetic diversity for breeders utilize for crop improvement. Approach and Results: The objective of this study was to evaluate the microsatellite markers (SSR) with 17 sugarcane accessions to access the genetic diversity and inter relationships in sugarcane. Genetic distances for SSR data (polymorphic fragments) were determined and relationships between samples were portrayed graphically in the form of a dendrogram and similarities are ranging from $36 \%$ to $100 \%$ were observed. The lowest genetic similarity of $36 \%$ was seen between sample 9 and 11 with other samples. These two genotypes differed from each other with only $68 \%$ similarity. Conclusion: Results illustrate that SSR markers could be useful for structuring the genetic diversity of collections according to geographical origin and ploidy level, assessment or formation of a core collection and especially construction of a genetic map.
\end{abstract}

Key words: SSR, UPGMA, Saccharum officinarum L, EST sequence, NTSYS-2.02

\section{INTRODUCTION}

Saccharum officinarum L. is a grass native of Southeast Asia (Souza et al., 2010). Sugarcane (Saccharum officinarum L.) is the main source for sugar production and plays a vital economic role in many tropical countries (Jangpromma et al., 2010). Sugarcane (Saccharum spp. hybrids) is a genetically complex crop of major economic importance in tropical and sub tropical countries (Singh et al., 2010). Saccharum, a complex genus characterized by high ploidy levels and composed of at least six distinct species-S. officinarum, $S$. barberi, $S$. sinensi, $S$. spontaneum, S. robustum and S. edule. Sugarcane is an industrial crop with acreage of about 4 million hectares and production to the tune of 300 million tones in India. The commercial sugarcane is no longer pure Saccharum officinarum but a species hybrid, complex polyploid with a large number of chromosomes. This does not offer a clean system for genetic manipulation through conventional means. As a result, inheritance of most of the characters of interest for varietal breeding is not thoroughly worked out, so much so that neither the number of genes nor the nature of gene action governing the expression of these traits is fully known. In addition to being a food crop, sugarcane is an efficient crop for producing fuel ethanol. In some parts of the world, alcohol has traditionally been produced as a byproduct of the sugar industry, through the fermentation of molasses and subsequent distilling (Usaborisut and Niyamapa, 2010).

Sugarcane is a complex aneu-polyploidy plant $(2 \mathrm{n}$ $=8 x$ or $10 x=100-130$ ) that propagates asexually through planting of vegetative cuttings (setts) of mature stalks (Grivet and Arruda, 2002). Microsatellite or Simple Sequence Repeats (SSRs) DNA markers are short DNA fragments that contain various numbers of tandem repeat units of di, tri, tetra or compositenucleotide motifs (Edwards et al., 1991; Polymeropoulos et al., 1991; Bruford and Wayne, 1993). SSR markers are useful for genotyping sugarcane because they are abundant, co-dominantly

\section{Corresponding Author: Ashutosh Pandey, Department of Biotechnology, Center for Biotechnology, School of Environmental Biology,} A.P.S. University, Rewa-486 003, Madhya Pradesh, India 
inherited and highly reproducible (Cordeiro et al., 2000; 2001). Since the beginning of the century, a high throughput molecular genotyping technology has been developed for sugarcane (Pan et al., 2003).

They are widely dispersed through eukaryotic genomes and are often highly polymorphic. These markers are one of the molecular tools of choice for biodiversity studies because of their high information content (Smith and Wayne, 1996). PCR amplification protocols used for microsatellites employ either unlabelled primer pairs or primer pairs with one of the primers being radiolabelled or fluorolabelled. Electrophoresis of unlabelled PCR products can be carried out on smaller vertical polyacrylamide gels or on horizontal agarose gels.

Microsatellites are co-dominant markers and the data generated are similar to those of allozymes, except that the number of alleles and heterozygosity revealed is almost always higher. Population genetic, parentage relatedness analysis can then be carried out. Slatkin (1995) and Goldstein et al. (1995) took advantage of our knowledge of the predominant mode of microsatellite evolution (i.e., stepwise mutation) to derive the measures of population subdivision and Average Genetic Distance (ASD). The strengths of microsatellites include the codominance of alleles, their high genomic abundance in eukaryotes and their random distribution throughout the genome, with preferential association in low-copy regions (Morgante et al., 2002). Because the technique is PCR-based, only low quantities of template DNA (10-100 ng per reaction) are required. Due to the use of long PCR primers, the reproducibility of microsatellites is high and analyses do not require high quality DNA.

Although microsatellite analysis is in principle a single-locus technique multiple microsatellites may be multiplexed during PCR or gel electrophoresis if the size ranges of the alleles of different loci do not overlap (Ghislain et al., 2004). This decreases significantly the analytical costs. Furthermore, the screening of microsatellite variation can be automated, if the use of automatic sequencers is an option.

Several studies to determine the genetic diversity within and between members of the Saccharum complex have been carried out using molecular systems such as AFLPs (Besse et al., 1998), RAPDs (Burner et al., 1997), isozymes (Glaszmann et al., 1989), RFLPs (Lu et al., 1994; Besse et al., 1997) and 5S rRNA intergenic spacer sequences (Pan et al., 2000). All these properties make EST-SSRs more popular among the existing markers for development of molecular maps or QTL analysis. Also they are being used widely in different plant species like rice (Kantety et al., 2002), bread wheat (Kantety et al., 2002; Gupta et al., 2003), Capsicum (Minamiyama et al., 2006; Portis et al., 2007), sugarcane (Cordeiro et al., 2001) and cotton (Park et al., 2005) for molecular mapping, genetic diversity, transferability. The SSR markers were useful for differentiating the genotypes and assessment of genetic relationships due to its reproducibility and to multiallelic, codominant and informative properties (Wong et al., 2009).

In present study we evaluate SSR markers to determining the extent of genetic diversity across the sugarcane accessions having their origin from different agroclimatic regions of Uttar Pradesh India. The main objective of the present study was to acknowledge whether polymorphism is sufficient to distinguish sugarcane accessions and to assess the patterns of genetic diversity among a selected group of Saccharum officinarum L. in order to provide more information to facilitate breeding programs and to surpass the productivity levels presented today.

\section{MATERIALS AND METHODS}

Plant material: Different genotypes of Saccharum officinarum were collected from different geographical regions of Uttar Pradesh, India (Table 1) were used in this study. The young leaf samples were taken from the two different crosses.

Isolation of Plant DNA from fresh tissue: Grind $0.5 \mathrm{gm}$ of leaf material in liquid nitrogen to fine power using pre chilled mortar and pestle. Transfer the powder to a $15 \mathrm{M}$ polypropylene centrifuge tube containing $5.0 \mathrm{~mL}$ of pre warmed extraction buffer use spatula to disperse the material completely.

Table 1: Sources and detail of Saccharum officinarum L. samples

\begin{tabular}{llll}
\hline & Geographical location & & RNA/DNA \\
Culture code & of Uttar Pradesh & Origin & concentration \\
\hline BICSO-1 & Meerut & Leaves & 1.67 \\
BICSO-2 & Ghaziabad & Leaves & 1.53 \\
BICSO-3 & Lucknow & Leaves & 1.89 \\
BICSO-4 & Faizabad & Leaves & 2.10 \\
BICSO-5 & Jhansi & Leaves & 1.47 \\
BICSO-6 & Aligarh & Leaves & 1.65 \\
BICSO-7 & Agra & Leaves & 1.63 \\
BICSO-8 & Saharanpur & Leaves & 1.76 \\
BICSO-9 & Kanpur & Leaves & 1.59 \\
BICSO-10 & Sultanpur & Leaves & 1.87 \\
BICSO-11 & Bareilly & Leaves & 1.90 \\
BICSO-12 & Sitapur & Leaves & 1.59 \\
BICSO-13 & Raibareli & Leaves & 1.97 \\
BICSO-14 & Hamirpur & Leaves & 1.84 \\
BICSO-15 & Ballia & Leaves & 1.59 \\
BICSO-16 & Maharajganj & Leaves & 1.70 \\
BICSO-17 & Pilibhit & Leaves & 1.85 \\
\hline & & &
\end{tabular}


OnLine J. Biol. Sci., 11 (4): 105-111, 2011

Table 2: Characteristics of 20 EST-derived SSRs for Saccharum officinarum L

\begin{tabular}{llllll}
\hline & Nucleotide sequence (5'-3') & & & \\
Primers code & Forward Primer & Reverse Primer & GC\% & Tm $\left({ }^{\circ}\right.$ C) & $\begin{array}{l}\text { Product } \\
\text { size }(\mathrm{bp})\end{array}$ \\
\hline AP-SSR01 & ATTTCTCTTCCACCCATCACA & ATATGCTGGCCTACCACTACCA & 45 & 60.74 & 277 \\
AP-SSR02 & CCATTCCTGTGCTCTGCC & AGACGATAACGCATGATTTGTG & 50 & 60.19 & 397 \\
AP-SSR03 & TGGAAGTATGTGAGCAATCTGG & CTAATCGAAGGACAATGGAACC & 45 & 59.98 & 217 \\
AP-SSR04 & CTGGTTTGCACATTGATCCTTA & TTCGTGGCATGGAATCATATAG & 41 & 59.99 & 384 \\
AP-SSR05 & AGGCTTGGTTGAGATGTATGGT & ATTGTTGGAACCTGGAAGTGAT & 43 & 59.81 & 295 \\
AP-SSR06 & TGAGAATGATGAGCACAAGTGA & GAAAGATGGACAGACATGCAGA & 43 & 59.85 & 105 \\
AP-SSR07 & CCACTTGCTTCTTGACCTTCTT & CACGGACTTGATCCAAAATGTA & 44 & 59.89 & 256 \\
AP-SSR08 & AGCTGACCTTATTGCCATGAAT & TCACTTGTGCTCATCATTCTCA & 41 & 59.71 & 385 \\
AP-SSR09 & GCTGACATGGTCTTGGGTTAT & GAGGATCTGGCTTCACAAATC & 46 & 60.16 & 372 \\
AP-SSR10 & CGACTCCCTCATTTGAAGTCC & AAGAAGGTCAAGAAGCAAGTGG & 48 & 59.96 & 355 \\
AP-SSR11 & ATTTTCTCTTCCACCCATCACA & ATATGCTGGCCTACCACTACCA & 46 & 60.74 & 277 \\
AP-SSR12 & TTCGGTGGTGAAATTAGGAACT & CGTCGAAAGATTTGATGTGATG & 41 & 60.18 & 243 \\
AP-SSR13 & GGAAAATGTCTCGCTGCTACTT & AGTTCCTAATTTCACCACCGAA & 43 & 59.89 & 151 \\
AP-SSR14 & CACAAATCATGCGTTATCGTCT & CAAGAACAGAGCTGCCTAAACA & 43 & 59.85 & 250 \\
AP-SSR15 & GTCTCGTCCGTATTGCCTCTA & ACGCATGATTTGTGTGTACCAT & 47 & 59.76 & 334 \\
\hline
\end{tabular}

Incubate samples at $60^{\circ} \mathrm{C}$ for 30 min with accessional mixing by gentle swirling. Add $3 \mathrm{~mL}$ Chloroform, isoamyl alcohol and mix by inversion to emulsify. Spin at $15,000 \mathrm{rpm}$ for $10 \mathrm{~min}$ at room temp. Remove the aqueous phase with a wide bore pipette, transfer to a clean tube and add $2 / 3$ volume of isopropanol and mix by quick gentle inversion. Spool DNA using a bent Pasteur pipette and transfer to another tube. Alternatively, if the DNA appears flocculent, centrifuge at $5000 \mathrm{rpm}$ for $2 \mathrm{~min}$ and gently pour off the supernatant. Wash the DNA pellet in 70\% ethanol (5-10 $\mathrm{mL}$ ) for $20 \mathrm{~min}$. Dry the pallet and dissolute in $500 \mu \mathrm{L}$ TE buffer. As a "classical" method we used a modified Cetyl Trimethyl Ammonium Bromide (CTAB) extraction protocol (Doyle and Doyle, 1990), which has been utilized with success in many plant species. This protocol is based on lysis and purification with CTAB that selectively precipitates DNA while maintaining the solubility of many polysaccharides.

Purification of DNA: Dissolve $10 \mathrm{mg}$ RNase in $1 \mathrm{~mL}$ of $10 \mathrm{mM}$ Tris HCL (pH-8.0) and $15 \mathrm{mM}$. $\mathrm{NaCl}$ in microfuge tube or (dissolve in $1 \mathrm{~mL}$ of $\mathrm{H}_{2} \mathrm{O}$ ). Heat the tube in boiling water bath at $100^{\circ} \mathrm{C}$ for $15 \mathrm{~min}$ to denature contaminating DNase. Cool slowly at room temperature. Add RNase $0.50 \mathrm{mg} \mathrm{mL}^{-1}$ or $5 \mu \mathrm{g} \mathrm{mL}^{-1}$ to DNA sample and incubate at $37^{\circ} \mathrm{C}$ for one hour. Add equal volume of phenol: Chloroform: Isoamyl alcohol (25:24:1) and mix. Spin at 11,000 rpm for $5 \mathrm{~min}$ at room temp take out the aqueous phase and transfer to a fresh microfuge tube. Extract twice with equal volume of Phenol: Chloroform: isoamyl alcohol (25:24:1), Centrifuge and take out the aqueous phase. Add 0:1 volume of $3 \mathrm{M}$ sodium acetate $(\mathrm{pH} 4.8)$ and mix properly. Add 2.5 times absolute alcohol mix by quick gentle diversion to precipitate the DNA. Pellet the DNA by centrifugation at $10,000 \mathrm{rpm}$ for $5 \mathrm{~min}$. in a microfuge. Decant supernatant carefully, wash the pellet with $70 \%$ ethanol and air dry to dissolute DNA in 50-100 $\mu \mathrm{L}$ TE Buffer.

PCR amplification and gel electrophoresis: Fifteen SSR primer pairs were used to determine the diversity were developed at School of Environmental Biology, A.P.S. University, Rewa, MP, India. These sequences of oligonucleotide were synthesized (Table 2). PCR reaction was carried out in a total of $25 \mu \mathrm{l}$ volume containing $10 \mathrm{ng}$ template DNA, $1.0 \mu \mathrm{l}$ of each forward and reverse primers, $100 \mathrm{mM}$ of dNTPs, $0.5 \mathrm{U}$ of Taq DNA polymerase, $1.0 \mu \mathrm{L}$ of $10 \mathrm{X}$ PCR buffer and 2.5 $\mathrm{mM}$ of $\mathrm{MgCl}_{2}$. Amplification condition was same as described by Williams et al. (1990) were performed in a Eppendorf master cycler (Eppendorf) with following conditions: initial denaturation at $94^{\circ} \mathrm{C}$ for $5 \mathrm{~min}$ followed by 25 cycles amplifications. Each amplification cycle was initially at $94^{\circ} \mathrm{C}$ for $1 \mathrm{~min}$ fallowed by annealing temperature (Ta) for $1 \mathrm{~min}$ and then $72^{\circ} \mathrm{C}$ for $2 \mathrm{~min}$; final extension at $72^{\circ} \mathrm{C}$ for $7 \mathrm{~min}$ was allowed. The amplified products were stored at $4^{\circ} \mathrm{C}$. The amplified products were separated by denaturing polyacrylamide gel electrophoresis $(8 \%)$ in $0.5 \mathrm{X}$ TBE buffer. Visualized of the bands was done by $0.5 \mu \mathrm{g} \mathrm{mL}^{-1}$ ethidium bromide staining and image were taken under UV light for further study.

\section{RESULTS}

In present study 15 primer pairs were designed, synthesised and tested against Saccharum officinarum. Out of these 5 primers AP-SSR03, AP-SSR05, APSSR07, AP-SSR011 and AP- SSR13 show polymorphism and produced 148 scorable polymorphic bands for high and low sugar lines. Among 15 markers developed, ten were monomorphic and five were polymorphic used to analyze genetic variability. 


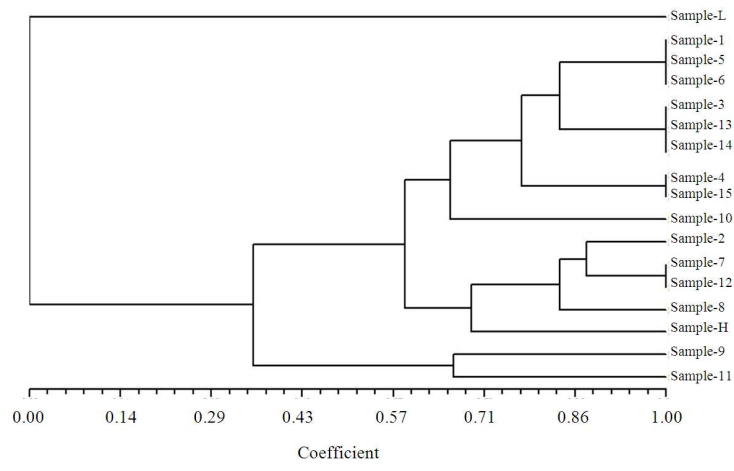

Fig. 1: Dendrogram generated from 148 SSR fragments for analysis of genetic relationships in Saccharum officinarum $\mathrm{L}$

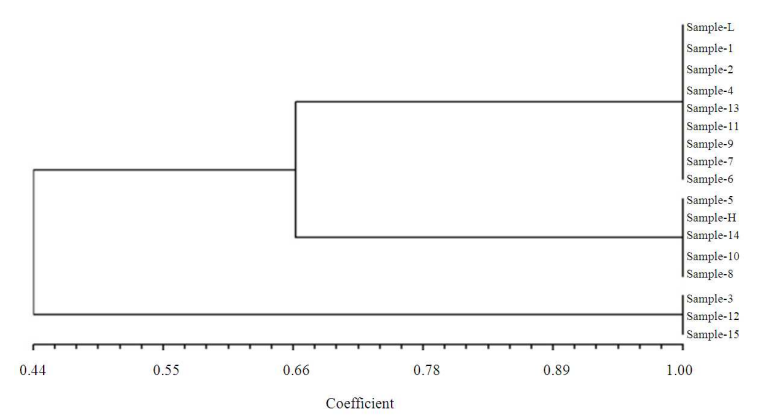

Fig. 2: Dendrogram derived from polymorphic DNA analysis of 17 Sugarcane samples with SSR primers by UPGMA. The bottom scale is the percentage of Jaccard's similarity coefficient

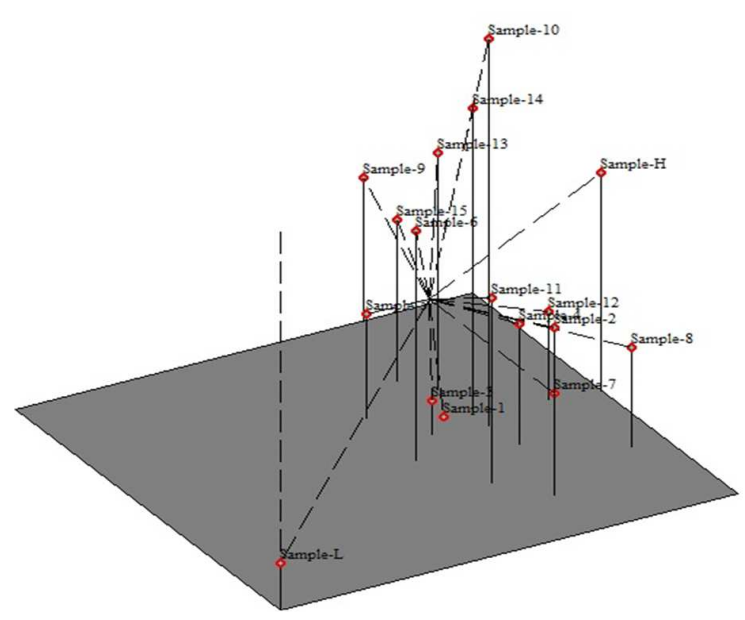

Fig. 3: Genetic diversity pattern among 17 genotypes based on SSR polymorphism depicted by principal coordinate analysis developed by NTSYS 2.02e software using SSR data obtained
All primers amplified polymorphic fragments, with a number of amplicons varying from 27 (AP-SSR07) to 12 (AP- SSR13) fragments per reaction, with sizes varying from $\sim 200 \mathrm{bp}$ to $\sim 2.0 \mathrm{~kb}$ while single fragments 275, 105, 372 bp by AP-SSR01, AP-SSR06 and APSSR09 respectively. Single fragments producing primers can be used as a marker for identification but primers produced polymorphic bands specific to set of genotypes and thus they may not have any diagnostic value in varietal identification.

Although none of the individual primers were enough to differentiate all the samples, highly polymorphic profiles were obtained from the sugarcane microsatellite primers AP-SSR07 and AP-SSR13 while ten primer pairs (AP-SSR01, AP-SSR02, AP-SSR04, AP-SSR06, AP-SSR08, AP-SSR09, AP-SSR10, APSSR12, AP-SSR14 and AP-SSR15) were found to be monomorphic. The banding pattern of the primers is shown in Fig. 1. Therefore, it may be concluded from the present results that SSRs can be used for identification of genetic diversity and the relationship between the low and high sugar content Saccharum officinarum. The molecular weight of the bands in each lane was taken for analysis for the presence and absence of the segregating bands for sugar trait in genotypes. Presence and absence of DNA band were representing by 1 and 0 respectively and the distance matrix were used to construct phylogenetic tree based on a parsimony criterion by using Un-weighted PairGroup Arithmetic Mean (UPGMA) programme contained in the software package NTSYS-2.02e (Rohlf, 1994). The dendrogram (Fig. 1) derived from SSR fingerprints clearly showed the existence of three major divergent groups with $36-100 \%$ similarities. Among the samples tested, high sugar content sample is $40 \%$ similar with sample 1, 5 and 6,50\% with sample 3, 4, 13, 14 and $15,70 \%$ with sample 7 and 12 while sample 2, 8, 9 and 11 are $60,77,33$ and $22 \%$ respectively.

Another dendrogram (Fig. 2) derived from monomorphic fragments revealed the polymorphism and clustered in two groups. Major group I consist two subgroups with $67 \%$ genetic similarity while within subgroups samples are $100 \%$ similar consisting sampleL, 2, 4, 6, 13, 11, 9, 7 and Sample-5, H, 14, 10, 8 in another subgroup. Sample-3, 15, 12 lies in a separate group but only $44 \%$ is similar with other samples. In present study we included low sugar content sample (sample-L) and high sugar content sample (sample-H), both are very similar with $67 \%$ similarity coefficient. For ease of interpretation, the genetic similarity values for SSR between pairs of genotypes were subjected to Principal Coordinate (PCoA) analysis to obtain graphical representations of the relationships between the 17 genotypes and develop a 3D distribution (Fig. 3) 
of samples on the basis of polymorphic band produced by 180 fragments of SSR markers of sugarcane genotypes. The PCoA revealed a pattern in which the individuals were assigned separately and 3D PCoA provided a better graphical illustration and a clear separation of samples on the basis of genetic characters.

\section{DISCUSSION}

Analyses of genetic variability using SSR markers have shown effective to detect polymorphism in several species of sugarcane. Studies with EST-SSR have shown great potential to generate polymorphism (Pinto et al., 2004, 2006), which allows organization of the genetic variability in germplasm banks. Using ESTSSR, Pinto et al. (2006) reported an average genetic similarity of 0.62 in 13 accessions analyzed. Alwala et al. (2006) using TRAP and AFLP markers detected a genetic similarity of 0.75 and 0.76 , respectively. These results suggest that genotypes of sugarcane have a high similarity of allele, which could result in lower genetic gain for selection. In terms of the discovery of SSR, development of functional primer pairs and polymorphism detection EST-SSR markers are less efficient than DNA-derived SSRs, however these caveats are balanced by the relatively low expense with EST-SSR development as a product of a genomic database (Faville et al., 2004).

The analysis of variations in SSR fragments provides an effective tool for examining diversity to improve plant breeding strategies. Identifying useful SSRs is critical but in sugarcane this can be a lengthy and difficult process due to their abundance and the complexity of the sugarcane genome. The amplification characteristics of 15 designed SSRs were evaluated. The number of alleles observed varied from 1 to 8 with AP-SSR07 and AP- SSR13 primer pairs found to be highly polymorphic. Twenty five SSRs were selected and used to examine diversity among a selection of sugarcane varieties that are either grown commercially or used in the breeding programs. The variation accounted for in between sample comparisons was low, however, significant differences were observed between sample-2, 8, 9, 10 and 11. Cluster analysis showed some general groups of varieties which for some varieties were consistent with pedigree information and in some cases were not. The information generated from this study will prove valuable in the selection of SSRs for future studies on sugarcane genetic diversity. The data presented will provide a useful guide in planning crosses for the maintenance and improvement of genetic diversity in sugarcane cultivars (Glynn et al., 2009).
The high level of polymorphism detected by SSR markers were expected due to the use of the external Indian sugarcane varieties and also due to allopolyploidy nature, generally attributed to the interspecific hybridization crosses used during breeding programs that generated the actual breeding accessions. The nature of SSR, targeting regions especially rich in microsatellites may also justify the higher level of polymorphism, since those regions are known to accumulate a larger number of mutations in DNA fragment during replication and unequal crossing-over. The complexity of the sugarcane genome and the abundance of SSRs make this a priority. Some 2005 clusters containing SSRs were identified from the analysis of the 237, 954 sugarcane ESTs (Pinto et al., 2004) in addition, 259 SSRs were developed through the International Consortium of Sugarcane Biotechnology (ICSB; (Cordeiro et al., 2000) and are available to ICSB members. Genomic SSRs have been shown to produce a greater number of alleles and higher PIC values than those from EST derived SSRs in sugarcane (Pinto et al., 2006). Marconi et al. (2011) developed EST-SSR functional markers for gene mapping and genetic diversity studies in sugarcane. These EST-SSR markers presented in this work can be efficiently used for genetic mapping studies of segregating sugarcane populations. The high Polymorphism Information Content (PIC) and Discriminant Power (DP) presented facilitate the QTL identification and marker-assisted selection due the association with functional regions of the genome became an important tool for the sugarcane breeding program.

Genetic distances for SSR data were determined and relationships between samples were portrayed graphically in the form of a dendrogram and similarities are ranging from $36 \%$ to $100 \%$ were observed among the 17 sugarcane samples while sample-L is completely away from the line (Fig. 1). The lowest genetic similarity of $36 \%$ was seen between sample-9 and 11 with other samples. These two genotypes differed from each other with only $68 \%$ similarity. SSR fingerprints can help sugarcane breeders to clarify the genetic pedigree of commercial sugarcane varieties and evaluate the efficiency of breeding methods.

Though there has been great advancement in the marker technology with the advent of different DNA markers, still SSR is quite convenient to apply provided the problem of reproducibility is minimized. The only option left over is to validate the SSR based assessment of genetic diversity by using maximum number of primers for the samples provided. In this study, SSR was carried out in order to determine the variability of 17 different genotypes. Considerable genetic diversity was observed among Saccharum officinarum. 


\section{CONCLUSION}

These results illustrate that SSR markers could be useful for structuring the genetic diversity of collections according to geographical origin and ploidy level, assessment or formation of a core collection and especially construction of a genetic map.

\section{ACKNOWLEDGEMENT}

Authors are thankful to head of A.P.S. University, Rewa, Madhya Pradesh, India for providing necessary research facilities and research grant in the form of ICAR-SRF.

\section{REFERENCES}

Alwala, S., C.A. Kimbeng, K.A. Gravois and K.P. Bischoff, 2006. TRAP, a new tool for sugarcane breeding comparison with AFLP and coefficient of parentage. J. Am. Soc. Sugar Cane Tech., 26: 62-86.

Besse, P., C.L. Mcintyre, D.M. Burner and C.G. Dealmeida, 1997. Using genomic slot blot hybridization to assess intergeneric Saccharum Erianthus hybrids (Andropogoneae-Saccharinae). Genome, 40: 428-432. DOI: 10.1139/g97-057

Besse, P., G. Taylor, B. Carroll, N. Berding, D. Burner and C.L. McIntrye, 1998. Assessing genetic diversity in a sugarcane germplasm collection using an automated AFLP analysis. Genetica, 104: 143-153. DOI: 10.1023/A:1003436403678

Bruford, M.W. and R.K. Wayne, 1993. Microsatellites and their application to population genetic studies. Cur. Opi. Gen. Dev., 3: 939-943. DOI: 10.1016/0959-437X(93)90017-J

Burner, D.M., Y.B. Pan and R.D. Webster, 1997. Genetic diversity of North American and Old World Saccharum assessed by RAPD analysis. Gen. Res. Crop Evo., 44: 235-240. DOI: 10.1023/A:1008631731506

Cordeiro, G.M., G.O. Taylor and R.J. Henry, 2000. Characterisation of microsatellite markers from sugarcane (Saccharum sp.), a highly polyploid species. Plant Sci., 155: 161-168. PMID: 10814819

Cordeiro, G.M., R. Casu, C.L. McIntyre, J.M. Manners and R.J. Henry, 2001. Microsatellite markers from sugarcane (Saccharum spp.) ESTs cross transferable to Erianthus and Sorghum. Plant Sci., 160: 1115-1123. PMID: 11337068

Doyle, J.J. and J.L. Doyle, 1990. Isolation of plant DNA from fresh tissue. Focus, 12: 13-15.
Edwards, A., A. Civitello, H.A. Hammond and C.T. Caskey. 1991. DNA Typing and Genetic Mapping with Trimeric and Tetrameric Tandem Repeats. Am. J. Human Gen., 49: 746-756.

Faville, M.J., A.C. Vecchies, M. Schreiber, M.C. Drayton and L.J. Hughes et al., 2004. Functionally associated molecular genetic marker map construction in perennial ryegrass (Lolium perenne L.). Tag Theo. Applied Gen., 110: 12-32. DOI: 10.1007/s00122-004-1785-7

Ghislain, M., D.M. Spooner, F. Rodriguez, F. Villamon and C. Nunez et al., 2004. Selection of highly informative and user-friendly microsatellites (SSRs) for genotyping of cultivated potato. Theo. Applied Gen., 108: 881-890.

Glaszmann, J.C., A. Fautret, J.L. Noyer, P. Feldmann and C. Lanaud, 1989. Biochemical genetic markers in sugarcane. Theo. Applied Gen., 78: 537-543. DOI: $10.1007 / \mathrm{BF} 00290839$

Glynn, N.C., K. McCorkle and J.C. Comstock, 2009. Diversity among mainland USA sugarcane cultivars examined by SSR genotyping. J. Am. Soc. Sugar Cane Technol., 29: 36-52.

Goldstein, D.B., A.R. Linares, L.L. Cavalli-Sforza and M.W. Feldamn, 1995. An evaluation of genetic distances for use with microsatellite loci. Genetics, 139: 463-471.

Grivet, L. and P. Arruda, 2002. Sugarcane Genomics: Depicting the Complex Genome of an Important Tropical Crop. Curr. Opion. Plant Biol., 5: 122127. DOI: 10.1016/S1369-5266(02)00234-0

Gupta, P.K., S. Rustgi, S. Sharma, R. Singh, N. Kumar and H.S. Balyan, 2003. Transferable EST-SSR markers for the study of polymorphism and genetic diversity in bread wheat. Mol. Gen. and Geno., 270: 315-323. DOI: 10.1007/s00438-003-0921-4

Jangpromma, N., S. Kitthaisong, K. Lomthaisong, S. Daduang, P. Jaisil and S. Thammasirirak 2010. A Proteomics Analysis of Drought Stress-Responsive Proteins as Biomarker for Drought-Tolerant Sugarcane Cultivars. Am. J. Agric. Biol. Sci., 6: 89-102. DOI: DOI: 10.3844/ajbbsp.2010.89.102

Kantety, R.V., M.L. Rota, D.E. Matthews and M.E. Sorrells, 2002. Data mining for simple sequence repeats in expressed sequence tags from barely, maize, rice, sorghum and wheat. Plant Mol. Biol., 48: 501-510.

Lu, Y.H., A. D'Hont, D.I.T. Walker, P.S. Rao, P. Feldmann and J.C. Glaszmann, 1994. Relationships among ancestral species of sugarcane revealed with RFLP using single copy maize nuclear probes. Euphytica, 78: 7-18. DOI: 10.1007/BF00021393 
Marconi, TG., E.A. Costa, R.C.A.N.H. Miranda, M.C. Mancini and C.B. Cardoso-Silva et al., 2011. Functional markers for gene mapping and genetic diversity studies in sugarcane. BMC Res. Notes, 4: 264-264. DOI: 10.1186/1756-0500-4-264

Minamiyama, Y., M. Tsuro and M. Hirai, 2006. An SSR-based linkage map of Capsicum annuum. Mol. Breed., 18: 157-169. DOI: 10.1007/s11032006-9024-3

Morgante, M., H. Hanafey and W. Powell, 2002. Microsatellites are preferentially associated with nonrepetitive DNA in plant genome. Nat. Gen. 30: 194-200. PMID: 11799393

Pan, Y.B., D.M. Burner and B.L. Legendre, 2000. An assessment of the phylogenetic relationship among sugarcane and related taxa based on the nucleotide sequence of 5S rRNA intergenic spacers. Genetica, 108: 285-295. DOI: 10.1023/A:1004191625603

Pan, Y.B., J.D. Miller, R.J. Schnell, E.P. Richard Jr. and Q. Wei, 2003. Application of microsatellite and rapd fingerprints in the florida sugarcane variety program. Proceedings of the 11th Plant Animal Genomes Conference, Jan. 11-15, Town and Country Convention Center, USA.

Park, Y.H., M.S. Alabady, M. Ulloa, B. Sickler and T.A. Wilkins et al., 2005. Genetic mapping of new cotton fiber loci using EST-derived microsatellites in an interspecific recombinant inbred line cotton population. Mol. Genet. Genomics., 274: 428-441. PMID: 16187061

Pinto, L.R., K.M. Oliveira, E.C. Ulian, A.A.F. Garcia and A.P.D. Souza, 2004. Survey in the sugarcane expressed sequence tag database (SUCEST) for simple sequence repeats. Genome, 47: 795-804. PMID: 15499394

Pinto, L.R., K.M. Oliveira, T. Marconi, A.A.F. Garcia and E.C. Ulian et al., 2006. Characterization of novel sugarcane expressed sequence tag microsatellites and their comparison with genomic SSRs. Plant Breed., 125: 378-384. DOI: 10.1111/j.1439-0523.2006.01227.x

Polymeropoulos, M.H., H. Xiao, D.S. Rath and C.R. Merril, 1991. Tetranucleotide Repeat Polymorphism at the Human Tyrosine hydroxylase gene (TH). Nucleic Acids Res., 19: 3753-3753. PMID: 1677184
Portis, E., I. Nagy, Z. Sasvari, A. Stagel and L. Barchi et al., 2007. The design of Capsicum spp. SSR assays via analysis of In silico DNA sequence and their potential utility for genetic mapping. Plant Sci., 172: 640-648. DOI: 10.1016/j.plantsci.2006.11.016

Rohlf, F.J. 1994. NTSYS-pc. Numerical Taxonomy and Multivariate Analysis System. 1st Edn., Exeter Software, New York, ISBN-10: 0925031224, pp: 7.

Singh, R.K., S.K. Mishra, S.P. Singh, N. Mishra and M.L. Sharma, 2010. Evaluation of microsatellite markers for genetic diversity analysis among sugarcane species and commercial hybrids. Aus. J. Crop Sci., 4: 115-124. DOI:

Slatkin, M., 1995. A measure of population subdivision based on microsatellite allele frequency. Genetics, 139: 457-462.

Smith, T.B. and R.K. Wayne, 1996. Molecular Genetic Approaches in Conservation. 1st Edn., Oxford University Press, New York, ISBN-10: 019509526X, pp: 483.

Souza, D.R.D., E. Stingel, L.C.D. Almeida, C.B. Munhae and A.J. Mayhe-Nunes et al., 2010. Ant diversity in a sugarcane culture without the use of straw burning in southeast, Sao Paulo, Brazil. Am. J. Agric. Biol. Sci., 5: 183-188. DOI: DOI: 10.3844/ajabssp.2010.183.188

Usaborisut, P. and T. Niyamapa, 2010. Effects of Machine-Induced Soil Compaction on Growth and Yield of Sugarcane. Am. J. Agric. Biol. Sci., 5: 269-273. DOI: DOI: 10.3844/ajabssp.2010.269.273

Williams, J.G.K., A.R. Kubelik, K.J. Livak, J.A. Rafalski and S.V. Tingey, 1990. DNA polymorphisms amplified by arbitrary primers are useful as genetic markers. Nuc.. Acids Res., 18: 6531-6535. DOI: 10.1093/nar/18.22.6531

Wong, S.C., P.H. Yiu, S.T.W. Bong, H.H. Lee, P.N.P. Neoh and A. Rajan, 2009. Analysis of Sarawak Bario Rice Diversity Using Microsatellite Markers. Am. J. Agric. Biol. Sci., 4: 298-304. DOI: 10.3844/ajabssp.2009.298.304 